\title{
Effect of Microplastics on Nasal and Intestinal Microbiota of High-Exposure Population: Protocol for an Observational Cross-sectional Study
}

\section{Xiyu Zhang}

Hospital of Chengdu University of Traditional Chinese Medicine

\section{Yuchi He}

Chengdu University of Traditional Chinese Medicine

\section{Ziyan Xie}

Chengdu University of Traditional Chinese Medicine

\section{Sihan Peng}

Chengdu University of Traditional Chinese Medicine

\section{Chunguang Xie}

Hospital of Chengdu University of Traditional Chinese Medicine

\section{Heting Wang}

University of Electronic Science and Technology of China

\section{Lu Liu}

Chengdu University of Traditional Chinese Medicine

\section{Jian Kang}

Hospital of Chengdu University of Traditional Chinese Medicine

\section{Haipo Yuan}

Hospital of Chengdu University of Traditional Chinese Medicine

Ya Liu ( $\square$ liuyaya918@163.com )

Hospital of Chengdu University of Traditional Chinese Medicine

\section{Study protocol}

Keywords: Microplastics, Environment, Nasal microbiota, Intestinal microbiota, 16S rRNA sequencing, 8700 LDIR laser infrared imaging

Posted Date: February 23rd, 2022

DOI: https://doi.org/10.21203/rs.3.rs-1374664/v1

License: (c) (1) This work is licensed under a Creative Commons Attribution 4.0 International License. Read Full License 


\section{Abstract}

\section{Background:}

Microplastics have the characteristics of small size, high specific area, strong ability to adsorb pollutants, and difficult to degrade. They have become a major global environmental problem that humans urgently need to address. A balanced micro-ecosystem is essential to human health. Animal studies have shown that long-term exposure to microplastics can change the characteristics of the microbiota in organisms, leading to respiratory, digestive, immune and other system diseases. However, the current research on microplastics is still dominated by animal experiments, and the impact of microplastics on human health is still in its infancy, so relevant research is urgently needed.

\section{Methods/Design:}

Sixty participants with high exposure to microplastics will come from a plastic factory in Chengdu, China. We will conduct 16 S rRNA sequencing and 8700 LDIR laser infrared imaging to the samples from the participants and from the environment. We will evaluate the health status of the participants through Short-Form Health Survey 36 (SF-36). For comparison, we will also collect samples and questionnaires from 60 volunteers from an area with good environmental quality in Chengdu. To find out the potential predictors and to access the difference between the groups, statistical analysis will be performed in the end.

\section{Discussion:}

The study will be the first observational cross-sectional study focusing on the effects of microplastics on nasal and intestinal microbiota of high-exposure population. The study is expected to provide reliable evidence to fill the gaps in the impact of microplastics on human health.

\section{Trial registration:}

This trial was registered with Chinese Clinical Trial Registry (ChiCTR2100049480) on August 2, 2021.

\section{Introduction}

The term "microplastic" was first used by Thompson in the literature in 2004 to describe tiny plastic particles existing marine environment(1). Microplastics is usually defined as plastic debris with size below $5 \mathrm{~mm}$ which comes from breaking of larger plastic objects (secondary microplastics) and tiny plastic particles (primary plastics) directly released by human activities(2). Only the year of 2018, the total plastics released by the whole world was up to 359 million tons(3). And it's not the first problem caused by the production, apply and consumption of plastics. In fact, it has caused thousands of major environment problems since 1950s. As a result of wild use of plastics, microplastics now is ubiquitous. The presences of microplastics have been reported in ocean, freshwater, food, outdoor and indoor air, soil, and even the South Pole(4-8). 
Researchers found out that the nasal cavity is the key part of the inhalation of microplastics in the air, and the intestine is the main enrichment part of the microplastics after ingestion(3). As is known to all, a balanced micro-ecosystem of the nasal cavity and intestines is essential to biological health.

Microplastics can enter human body by ingestion and inhalation and cause diseases. Studies indicated that worker who is in high exposure to microplastics such as polypropylene (PP) and Polyamide (PA) at work shows increased risk of lung diseases(9). What's more, the risk of polypropylene flocked workers having respiratory symptoms increased 3.6 times compared with the control group. And subtle or minor interstitial lung diseases were also found within those workers(10). According to some animal studies, long-term exposure to microplastics would alter the characteristics of microbiota inside, which may lead to diseases in respiratory, digestive, and immune system(11-13).

This study is designed to investigate how microplastics effect on nasal and intestinal microbiota of highexposure population (workers in plastic factory). The study is expected to provide reliable evidence, fill the gaps in the impact of microplastics on human health, and provide a strong basis for the improvement of relevant information on the toxic effects of microplastics on humans, and the establishment of standards for microplastics in the environment.

\section{Methods And Analysis}

\subsection{Study design}

This study is designed as a observational cross-sectional study and was developed according to the Strengthening the Reporting of Observational Studies in Epidemiology (STROBE) (the STROBE checklist is shown in Supplemental file 1). A flowchart of this trial procedure is shown in Figure 1. The nasal and intestinal secretions of subjects exposed to microplastics will be collected and analyzed to determine the characteristics of the nasal and intestinal flora under high exposure of microplastics. Participants will sign a written informed consent form (Figure 2, Supplemental file 2) and have a clear understanding of the purpose, study procedures and all potential risks related to the study.

\subsection{Study subject}

The study is conducted at a plastic factory in Chengdu, China, which mainly produces plastic products including polyethylene, polyvinyl chloride, polytetrafluoroethylene, polypropylene, and polystyrene. We will post recruitment information on intranet and bulletin boards of the factory. Volunteers should meet the inclusion and exclusioncriteria below to officially become a participant of the study (Inclusion and exclusion criteria for study enrollment detailed in Figure 3).

Members of the research group (ZXY, PSH, WHT, LY) will be involved in recruiting participants, all of whom are medical staff with physician certificates and will receive the necessary training to communicate with people and collect samples. The recruitment period is from July 2021 to July 2022, and each group is expected to recruit 60 participants(14). All information and data of participants will be confidential. Only members of the research group and principal investigator can have access to them. 
After the late data entry is completed, participants can log on to the website (http://www.medresman.org.cn/login.aspx ) to query the details.

To clarify the characteristics of the microbiota of the sduty group, we will also collect samples and questionnaires from 60 volunteers from areas with good environmental quality in Chengdu. These volunteers will also meet the inclusion and exclusion criteria except that they have been living or working continuously for $\geq 6$ months and the daily time is $\geq 8$ hours per day within 1 kilometers from the center of the Huanhuaxi Park. Huanhuaxi Park is the largest open city forest and wetland park (32.32 hectares) in Chengdu, China, with significantly better air quality index than other regions of Chengdu.

\subsection{Sample size}

We referred to previous article(14) and considered the lost-to-follow-up rate of approximately $15 \%$. Based on this, We estimated that a sample of 60 participants is enough to explore the microbiota characteristics for the study.

\subsection{Data and sample collection}

All the data of the study will be collected and managed by Chinese Clinical Management Public Platform (http://www.medresman.org.cn/login.aspx) which records the management process of clinical trials, baseline data of subjects recorded during the trial, result data and other relevant data based on internet and upload them to the central database for preservation and management. The data of this study can only be accessed and operated by the research team. Once the data are entered and stored, any changes made to the data will be automatically displayed and tracked. The public can view the related public information through "Public Browsing" when the enrollment is finished, but they will not be able to trace any personal information of the participants.

All members who collect samples and data will receive necessary training to make sure the accuracy of data. Anthropometric characteristics (including height without shoes, fasting weight in the morning) and Short-Form Health Survey 36 (SF-36)(15) will be finished right after the enrollment. And we will recheck all the collected data at the end of the collecting. If there's any missing data (such as height of some participants, unfinished questionnaires and so on), we will contact the certain participant to fix it. If there're some participants with missing data we can't reach, we will record the number of participants with missing data for each variable of interest.

Researchers must wear disposable sterile gloves when collecting biological samples to avoid contacting with the actual sampling area and its surrounding area. If the glove is contaminated, it must be changed immediately. The study involved the collection and storage of biological specimens. All biological specimens will be destroyed right after use.

\subsubsection{Collecting specimens of intestinal secretions}


Participants will receive necessary training on how to collect their own intestinal secretions after enrollment. They will receive prepared cryopreservation box, marked sterile feces collector and sterile glass vials containing $5 \mathrm{ml}$ of $75 \%$ medical alcohol in advance. Among them, samples in feces collector will be used for $16 \mathrm{~S}$ rRNA sequencing, and samples in glass vials will be used for microplastic composition analysis.

Intestinal sections of each participant will be collected by sterile spoon (contained within the feces collector) and placed in the sterile feces' collector and sterile glass vials respectively. These samples will be asked to store in the cryopreservation box immediately. And every participant should inform the research team right after their collecting. Members of our group will be responsible for transmitting those samples. We will put the sterile feces collector in liquid nitrogen for 4 hours and then transfer it to $-80^{\circ} \mathrm{C}$ for storage. And we will put sterile glass vials into $4^{\circ} \mathrm{C}$ to store.

\subsubsection{Collecting specimens of nasal secretions}

Researchers who are responsible for collecting nasal samples will receive necessary training on how to collect nasal secretion samples. The procedure we will perform is shown as below: first, gently rotate and wipe with a sterile cotton swab on the mucosal layer of the superior turbinate (about $2 \mathrm{~cm}$ ) of the nasal cavity 2-3 times with the help of nasal endoscope; second, withdraw the swab slowly and place it into a sterile cryopreservation tube immediately; third, combine the left and right nasal samples of each participant into one; last, put them in liquid nitrogen for 4 hours and then transfer to $-80{ }^{\circ} \mathrm{C}$ for storage.

\subsubsection{Collecting environmental specimens}

We will collect soil and air samples within 1 kilometers from the center of the Huanhuaxi Park and at the plastic factory. Samples will be collected at 5 spots including the east, west, south, north and center of the selected area (Figure 4$)$. The topsoil $(10 \mathrm{~cm})$ at 5 sampling points will be collected using a stainless steel sampling shovel(16-18). Those samples will be mixed uniformly to be one composite sample and wrapped by aluminum foil into a sampling bag(18). Before testing, we will place the samples in a clean, dry, dark place and store them at a low temperature. Outdoor air will be collected through an active suction sampler (MiniVolTM, Airmetrics, USA), which will be on for 6-8 hours at an adult's breathing height $(1.5 \mathrm{~m})$ at $5 \mathrm{~L} / \mathrm{min}(19,20)$.

\subsection{Outcome measures}

We will collect samples and questionnaires right after the enrollment. Every participant will take assessment only one time.

\subsubsection{Primary outcome measures}

Diversity of nasal and intestinal microbiota analyzed by 16S rRNA sequencing (Illumina Hiseq 2500 sequencing platform, Biomarker Technologies Corporation, Beijing, China). 
Microplastics from intestinal secretions and environmental samples analyzed by 8700 LDIR laser infrared imaging (Agilent Technologies Co., Ltd, USA).

\subsubsection{Secondary outcome measure}

Health status of participants evaluated by SF-36 questionnaire, which includes physical functioning, physical role, physical pain, general health, vitality, social functioning, emotional role, and mental health. After the questionnaire is completed, a COOP/WONCA Chart will be generated. We will also investigate the anthropometric characteristics of the study group, which can include height without shoes, fasting weight in the morning and so on.

\subsection{Data management and monitoring}

Members of the research group will be responsible for collecting data. One statistician and one medical staff with physician certificate will be responsible for monitoring data. We will collect Case Report Forms which includes the basic information of participants and SF-36 questionnaire. Two members of the research group (HYC, XZY) will enter collected information into the Chinese Clinical Trial Management Public Platform under confidential condition. The hard copy records will be preserved at a locked office. No one will be able to change or use the hard copy and electronic data without the authorization of our group.

\section{Statistical Methods}

We will analyze quantitative data (age, working year, score of questionnaires, etc.) to obtain each variable's average (arithmetic mean, geometric mean or median up to the distribution), standard deviation, inter-quartile range, etc. So that we can find out if background characteristics of the study group would be different from the volunteer group around the Huanhuaxi park. We will also analyze qualitative data for variables such as gender distribution and proportion of each microbe species. And we will identify the core species based on the proportion above. We will compare the dichotomous variables between the two groups or among participants in the study group with or without certain microbe species using chi-square test or Fisher's exact test if the theoretical frequency is $<1$. We will compare the continuous variables between the two groups using t-test or Wilcoxon signed-rank test if the distribution is not normal. We will also perform analysis to check the microbiota alteration among the three subgroups and compare it with the control group. We will perform multiple logistic regression for categorical variables and multiple linear regression for quantitative data to find out the potential predictors. We're expecting to figure out how high exposure to microplastics would alter the structure and characteristics of microbiota. SPSS 24.0 software will be used to perform statistical analysis. $P<0.05$ will be considered as statistically significantly.

\section{Discussion}


Microplastics is one of those inhalable particles which can enter human body through respiratory tract. Digestive tract is considered as another major path for microplastics to enter human body(21), in which gut is the most gathering part. As the most intelligent creature in this planet, human beings can dominate the food chain, but also undergo much greater risks to intake more microplastic because of bioconcentration $(22,23)$. It's estimated that the amount of microplastics consumed by one American resident per year is 39,000-52,000(24). United European Gastroenterology Week in 2018 indicates that human feces contain microplastics with an average of 20 microplastics $/ 10 \mathrm{~g}$ feces and a size of 50-500 $\mu \mathrm{m}$ The current animal studies shows that exposure to microplastics can lead to the imbalance of intestinal microbiota of mice $(11,13,25-28)$. Specifically, firmicutes increased significantly, while Bacteroidetes and Proteobacteria decreased significantly in those mice. We all know that it's crucial to maintain the balance of microecosystem of nasal and intestinal cavity for human health. More and more researchers are starting to realize the harmfulness of microplastics.

The current study will be the first observational cross-sectional study focusing on the effect of microplastics on high-exposure population, which is expected to fill the gaps in the impact of microplastics on human health and explore the characteristics of nasal and intestinal microbiota of highexposure population. However, there're still some limitations of this study. First, participants in study group will all come from one factory, which may not be able to represent the whole high-exposure population. Second, we collect environmental samples from multiple locations, yet the current collection standards are not completely unified, which may make our regional samples results have a certain degree of deviation.

\section{Declarations}

\section{Acknowledgments}

We would like to thank all participants for their support and contributions to this study. We also thank Cao Zhiqing, a statistician from Chengdu University of Traditional Chinese Medicine, for his guidance on this study.

\section{Trial registration}

This trial was registered with Chinese Clinical Trial Registry (ChiCTR2100049480) on August 2, 2021.

Trial status: Recruitment began in August 2021, and the data analysis is expected to be completed in August 2022.

\section{Ethical approvals and consent to participate}

The Medical Ethics Committee of Hospital of Chengdu University of Traditional Chinese Medicine has approved this trial and the approval number is $2021 \mathrm{KL}-065$. All participants in the study group and volunteers in the control group will give their written informed consent. The study protocol will be implemented in accordance with the guidelines of the Declaration of Helsinki. 


\section{Consent for publication}

Not applicable.

\section{Patient and public involvement}

Patients or the public were not involved in the design, or conduct, or reporting, or dissemination plans of our research.

\section{Dissemination of trial findings}

The results will be communicated locally and internationally as conference papers and journal articles.

\section{Availability of data and materials}

Data sharing is not applicable to this article as no datasets are reported. Availability of datasets generated in the study will be included in papers reporting study outcomes.

\section{Authors' contributions}

LY designed the study protocol and contributed to the subject recruitment. ZXY designed and wrote the study protocol and will contribute to the data collection, laboratory measurement, and analysis. HYC contributed to wrote the study protocol and will assist in the data collection and analysis. XZY will perform laboratory measurements. PSH, WHT, and LL will perform statistical analysis. XCG contributed to the study design, revised and edited the manuscriptr. KJ will assist in subject recruitment and data collection. YHP will assist in subject recruitment and data collection. All authors read and approved the final manuscript.

\section{Funding}

This study was supported by the National Natural Science Foundation of China [82104813], General Program of China Postdoctoral Science Foundation [2020M673563XB], Chengdu University of Traditional Chinese Medicine Xinglin Scholars Scientific Research Fund [BSH2019024], and Science and Technology Development Fund of Affiliated Hospital of Chengdu University of Traditional Chinese Medicine [Y2019091 and Y2019120].

\section{Competing interests}

The authors declare that the research was conducted in the absence of any commercial or financial relationships that could be construed as a potential conflict of interest.

\section{References}


1. Thompson RC, Olsen Y, Mitchell RP, Davis A, Rowland SJ, John AW, et al. Lost at sea: where is all the plastic? Science. 2004;304(5672):838.

2. Weinstein JE, Crocker BK, Gray AD. From macroplastic to microplastic: Degradation of high-density polyethylene, polypropylene, and polystyrene in a salt marsh habitat. Environmental toxicology and chemistry. 2016;35(7):1632-40.

3. Hirt N, Body-Malapel M. Immunotoxicity and intestinal effects of nano- and microplastics: a review of the literature. Particle and fibre toxicology. 2020;17(1):57.

4. Eriksen M, Lebreton LC, Carson HS, Thiel M, Moore CJ, Borerro JC, et al. Plastic Pollution in the World's Oceans: More than 5 Trillion Plastic Pieces Weighing over 250,000 Tons Afloat at Sea. PLoS One. 2014;9(12):e111913.

5. Toussaint B, Raffael B, Angers-Loustau A, Gilliland D, Kestens V, Petrillo M, et al. Review of micro- and nanoplastic contamination in the food chain. Food additives \& contaminants Part A, Chemistry, analysis, control, exposure \& risk assessment. 2019;36(5):639-73.

6. Waring RH, Harris RM, Mitchell SC. Plastic contamination of the food chain: A threat to human health? Maturitas. 2018;115:64-8.

7. Guo JJ, Huang XP, Xiang L, Wang YZ, Li YW, Li H, et al. Source, migration and toxicology of microplastics in soil. Environment international. 2020;137:105263.

8. Bessa F, Ratcliffe N, Otero V, Sobral P, Marques JC, Waluda CM, et al. Microplastics in gentoo penguins from the Antarctic region. Scientific reports. 2019;9(1):14191.

9. Kern DG, Kuhn C, 3rd, Ely EW, Pransky GS, Mello CJ, Fraire AE, et al. Flock worker's lung: broadening the spectrum of clinicopathology, narrowing the spectrum of suspected etiologies. Chest. 2000;117(1):251-9.

10. Atis S, Tutluoglu B, Levent E, Ozturk C, Tunaci A, Sahin K, et al. The respiratory effects of occupational polypropylene flock exposure. The European respiratory journal. 2005;25(1):110-7.

11. Jin Y, Xia J, Pan Z, Yang J, Wang W, Fu Z. Polystyrene microplastics induce microbiota dysbiosis and inflammation in the gut of adult zebrafish. Environmental pollution (Barking, Essex : 1987). 2018;235:322-9.

12. Liu Z, Yu P, Cai M, Wu D, Zhang M, Chen M, et al. Effects of microplastics on the innate immunity and intestinal microflora of juvenile Eriocheir sinensis. The Science of the total environment. 2019;685:836-46.

13. Ju H, Zhu D, Qiao M. Effects of polyethylene microplastics on the gut microbial community, reproduction and avoidance behaviors of the soil springtail, Folsomia candida. Environmental pollution (Barking, Essex : 1987). 2019;247:890-7.

14. Mariani J, Favero C, Spinazzè A, Cavallo DM, Carugno M, Motta V, et al. Short-term particulate matter exposure influences nasal microbiota in a population of healthy subjects. Environmental research. 2018;162:119-26.

15. Tan ML, Wee HL, Lee J, Ma S, Heng D, Tai ES, et al. The Short Form 36 English and Chinese versions were equivalent in a multiethnic Asian population. Journal of clinical epidemiology. 2013;66(7):759- 
67.

16. Corradini F, Bartholomeus H, Huerta Lwanga E, Gertsen H, Geissen V. Predicting soil microplastic concentration using vis-NIR spectroscopy. The Science of the total environment. 2019;650(Pt 1):92232.

17. Beriot N, Peek J, Zornoza R, Geissen V, Huerta Lwanga E. Low density-microplastics detected in sheep faeces and soil: A case study from the intensive vegetable farming in Southeast Spain. The Science of the total environment. 2021;755(Pt 1):142653.

18. Du C, Liang H, Li Z, Gong J. Pollution Characteristics of Microplastics in Soils in Southeastern Suburbs of Baoding City, China. International journal of environmental research and public health. 2020;17(3).

19. Prata JC, Castro JL, da Costa JP, Duarte AC, Rocha-Santos T, Cerqueira M. The importance of contamination control in airborne fibers and microplastic sampling: Experiences from indoor and outdoor air sampling in Aveiro, Portugal. Marine pollution bulletin. 2020;159:111522.

20. Li Y, Shao L, Wang W, Zhang M, Feng X, Li W, et al. Airborne fiber particles: Types, size and concentration observed in Beijing. The Science of the total environment. 2020;705:135967.

21. Jambeck JR, Geyer R, Wilcox C, Siegler TR, Perryman M, Andrady A, et al. Marine pollution. Plastic waste inputs from land into the ocean. Science. 2015;347(6223):768-71.

22. Hidalgo-Ruz V, Gutow L, Thompson RC, Thiel M. Microplastics in the marine environment: a review of the methods used for identification and quantification. Environmental science \& technology. 2012;46(6):3060-75.

23. Smith M, Love DC, Rochman CM, Neff RA. Microplastics in Seafood and the Implications for Human Health. Current environmental health reports. 2018;5(3):375-86.

24. Cox KD, Covernton GA, Davies HL, Dower JF, Juanes F, Dudas SE. Human Consumption of Microplastics. Environmental science \& technology. 2019;53(12):7068-74.

25. Wan Z, Wang C, Zhou J, Shen M, Wang X, Fu Z, et al. Effects of polystyrene microplastics on the composition of the microbiome and metabolism in larval zebrafish. Chemosphere. 2019;217:646-58.

26. Qiao R, Deng Y, Zhang S, Wolosker MB, Zhu Q, Ren H, et al. Accumulation of different shapes of microplastics initiates intestinal injury and gut microbiota dysbiosis in the gut of zebrafish. Chemosphere. 2019;236:124334.

27. Lu L, Wan Z, Luo T, Fu Z, Jin Y. Polystyrene microplastics induce gut microbiota dysbiosis and hepatic lipid metabolism disorder in mice. The Science of the total environment. 2018;631-632:44958.

28. Wang HT, Ding J, Xiong C, Zhu D, Li G, Jia XY, et al. Exposure to microplastics lowers arsenic accumulation and alters gut bacterial communities of earthworm Metaphire californica. Environmental pollution (Barking, Essex : 1987). 2019;251:110-6.

\section{Figures}




\section{Figure 1}

Study process: flowchart of study procedure.

\section{Figure 2}

Informed consent form.

\section{Figure 3}

Inclusion and exclusion criteria for the study enrollment.

\section{Figure 4}

Map of sampled area. (A) Huanhuaxi Park, (B) Plastic factory.

\section{Supplementary Files}

This is a list of supplementary files associated with this preprint. Click to download.

- Supplementalfile1STROBEchecklistcrosssectional.docx

- Supplementalfile2InformedConsentForm.docx 